\title{
Efficacy and safety of turmeric and curcumin in lowering blood lipid levels in patients with cardiovascular risk factors: a meta-analysis of randomized controlled trials
}

Si Qin ${ }^{1}$, Lifan Huang ${ }^{1}$, Jiaojiao Gong ${ }^{1,2}$, Shasha Shen ${ }^{1,2}$, Juan Huang ${ }^{1,2}$, Hong Ren ${ }^{2}$ and Huaidong $\mathrm{Hu}^{1,2^{*}}$

\begin{abstract}
Background: Dyslipidemia is an important and common cardiovascular risk factor in the general population. The lipid-lowering effects of turmeric and curcumin are unconfirmed. We performed a meta-analysis to assess the efficacy and safety of turmeric and curcumin in lowering blood lipids in patients at risk of cardiovascular disease (CVD).

Methods: A comprehensive literature search was conducted on PubMed, Embase, Ovid, Medline and Cochrane Library databases to identify randomized controlled trials (published as of November 2016) that assessed the effect of turmeric and curcumin on blood lipid levels including total cholesterol (TC), low-density lipoprotein cholesterol (LDL-C), high-density lipoprotein cholesterol (HDL-C), and triglycerides (TG). Pooled standardized mean difference (SMD) with 95\% confidence interval (CI) was used to assess the effect.

Results: The analysis included 7 eligible studies (649 patients). Turmeric and curcumin significantly reduced serum LDL-C (SMD $=-0.340,95 \%$ confidence interval $[C 1]$ : -0.530 to $-0.150, P<0.0001)$ and TG $(S M D=-0.214$, $95 \% \mathrm{Cl}:-0.369$ to $-0.059, P=0.007$ ) levels as compared to those in the control group. These may be effective in lowering serum TC levels in patients with metabolic syndrome (MetS, SMD $=-0.934,95 \% \mathrm{Cl}:-1.289$ to -0.579 , $P<0.0001)$, and turmeric extract could possibly have a greater effect on reducing serum TC levels $(S M D=-0.584$, $95 \% \mathrm{Cl}:-0.980$ to $-0.188, P=0.004)$; however, the efficacy is yet to be confirmed. Serum HDL-C levels were not obviously improved. Turmeric and curcumin appeared safe, and no serious adverse events were reported in any of the included studies.

Conclusions: Turmeric and curcumin may protect patients at risk of CVD through improving serum lipid levels. Curcumin may be used as a well-tolerated dietary adjunct to conventional drugs. Further research is required to resolve uncertainties related to dosage form, dose and medication frequency of curcumin.
\end{abstract}

Keywords: Turmeric, Curcumin, Cholesterol, Cardiovascular risk, Triglycerides, Meta-analysis

\footnotetext{
* Correspondence: huhuaidong@sina.com

'Department of Clinical Nutrition, The Second Affiliated Hospital of Chongqing Medical University, No.76, Linjiang Road, Chongqing 400010, People's Republic of China

2Department of Infectious Diseases, Institute for Viral Hepatitis, Key Laboratory of Molecular Biology for Infectious Diseases (Ministry of Education), Institute for Viral Hepatitis, The Second Affiliated Hospital of Chongqing Medical University, Chongqing, People's Republic of China
} 


\section{Introduction}

Cardiovascular disease (CVD) is currently the leading cause of mortality worldwide [1]. Major cardiovascular risk factors include age, sex, hypertension, dyslipidemia, obesity, type 2 diabetes mellitus (T2DM), and metabolic syndrome (MetS). Dyslipidemia, hypertension, and insulin resistance, manifesting as T2DM and MetS, promote endothelial dysfunction and vascular inflammation leading to atherosclerosis-the main cause of CVD [2]. An epidemiological study showed that low circulating concentrations of low-density lipoprotein cholesterol (LDL-C) and triglycerides (TG) were associated with a low risk of CVD [3]. Therefore, treatment of dyslipidemia is critical for the prevention of CVD. Statins and fibrates are common lipid-modulating agents and a newer lipid-lowering agent, alirocumab (Praluent), has recently been approved for the treatment of dyslipidemia [2]. Statins are widely used despite their potential to cause serious adverse effects such as myopathies and hepatotoxicity [4, 5]. The estimated incidence of asymptomatic elevation in aminotransferases in patients on statin therapy is less than $2 \%$ [6] and the risk of developing rhabdomyolysis (a form of myopathy) is 3 per 100,0000 person-years (associated fatality rate: 10\%) [7]. Due to these concerns, it is crucial to focus efforts toward developing more effective drugs and to discover natural agents as alternatives to currently available treatments.

Turmeric (Curcuma longa), an Indian spice, is a yellow pigment that is used worldwide in cooking, cosmetics, dyes, and medicines [8]. It is worth noting that turmeric is a frequently used food additive in Southeast Asia, which improves color and flavor of food preparations. Curcumin (chemical name: diferuloylmethane) is an active component of turmeric [9], which has the capacity to interact with hundreds of molecular targets. Several studies have demonstrated the protective effects of curcumin against many chronic diseases, including various cancers, pulmonary disorders, and autoimmune diseases [10]. It has been shown to attenuate oxidative stress [11] and to exert a cardioprotective effect owing to its lipid-lowering properties [12-14]. However, some contradictory results have also been reported [15-21]. In a meta-analysis of 5 randomized controlled trials, overall effects in the entire study population as well as those in a subgroup analysis of subjects with cardiovascular risk factors failed to demonstrate a significant lipid-lowering effect of curcumin [22]. However, the studies included in this meta-analysis had limited sample sizes. Further, most of the randomized controlled trials that have reported positive effects of curcumin on blood lipid levels were published subsequent to the above-mentioned meta-analysis [23-28]. Nonetheless, conflicting reports exist in that some studies have reported promising effects [23-28], whereas others failed to demonstrate any significant effect [15]. Thus, we conducted a meta-analysis of published clinical trials to assess the efficacy and safety of turmeric and cucumin in lowering lipid levels in patients with risk factors for CVD.

\section{Methods \\ Literature search}

An online search was carried out for clinical studies published in English language in the following electronic databases: PubMed, Embase, Ovid, Medline and Cochrane Library. All studies published as of November 2016 were eligible for inclusion. Medical Subject Heading (MeSH) terms were used for PubMed, and comparable terms were used for other databases. The search terms were as follows: (curcuminoid OR curcumin OR curcuma OR turmeric OR curcuminoids) AND (hyperlipidemia OR hyperlipidemic OR hypolipidemic OR dyslipidemia OR dyslipidemic OR hypercholesterolemia OR hypercholesterolemic OR hypocholesterolemic OR "low-density lipoprotein" OR "high-density lipoprotein" OR cholesterol OR triglycerides OR hypertriglyceridemia OR hypotriglyceridemic). In addition, we used various synonyms.

\section{Inclusion and exclusion criteria}

Inclusion criteria for research articles included: (1) drug or placebo-controlled parallel randomized trials; (2) subjects with risk factors for CVD, for example, dyslipidemia, T2DM, MetS, hypertension, prediabetes, prehypertension, or obesity; (3) studies that used purified curcumin or a curcuminoid mixture, extracts with determined content of curcumin or curcuminoids, or turmeric powder, regardless of dosage and frequency (this inclusion criteria was established according to the meta-analysis [22] mentioned earlier and a review about the efficacy of turmeric/curcumin for alleviating the symptoms of arthritis [29]); (4) study duration $\geq 4$ weeks; and (5) studies that reported mean \pm standard deviation (SD), mean \pm standard error (SE) or median (range) of between-group differences between the experimental and control groups at baseline as well as at the end of the trial. Studies were excluded if they featured: (1) a crossover randomized trial design; (2) noncomparative data; (3) lack of outcome measures; and (4) duration of treatment $<4$ weeks.

\section{Efficacy measures}

Primary outcome measures included serum levels of LDL-C, high-density lipoprotein cholesterol (HDL-C), TG, and total cholesterol (TC). Treatment safety, measured by adverse effects due to turmeric and curcumin, was defined as a secondary outcome.

\section{Data extraction}

Two independent investigators (SQ and LFH) screened the titles and abstracts of articles initially retrieved on online search of databases, and extracted essential data 
from eligible full-text articles. Data on study design, patient characteristics, number of patients, treatment regimen, duration of treatment, year of publication, and daily dose of curcumin or turmeric powder were extracted, as were the mean \pm SD (or mean \pm SE) of all efficacy measures specified earlier. To control relative heterogeneity, only data of efficacy measures pertaining to the study period between 4 weeks and 3 months were extracted. For studies with missing data, authors were sent emails requesting details of these data.

\section{Study quality}

Risk of bias was assessed independently by 2 reviewers (SQ and LFH) using the Cochrane Handbook for Systematic Reviews of Interventions [30]. This tool allows for assessment of the study quality with respect to 6 domains: random sequence generation, allocation concealment, blinding, incomplete outcome data, selective reporting, and other bias. For each domain, the risk of bias was marked as low, unclear, or high.

\section{Statistical analysis}

The meta-analysis was conducted by using Stata version 12.0 (Stata Corporation, College Station, TX, USA). In this analysis, only continuous variables were extracted. If mean values and SD were unavailable, these were calculated from mean values and SE by using the following formulas: mean = mean post-treatment - mean pre-treatment; $\mathrm{SD}=\mathrm{SE} \times$ square root $\mathrm{n}$ ( $\mathrm{n}$ : number of participants) and $\mathrm{SD}=$ square root $\left[(\mathrm{SD} \text { pre-treatment })^{2}+(\mathrm{SD} \text { post-treatment })^{2}-\right.$ $(2 R \times \mathrm{SD}$ pre-treatment $\times \mathrm{SD}$ post-treatment) $]$, assuming a correlation coefficient $(R)=0.5$ [31]. With use of relevant formulae [32], these values were also calculated from medians and ranges. Because, one of the included studies [23] did not report the unit of outcomes, standardized mean difference (SMD) with 95\% confidence interval (CI) was used to present the results of the metaanalysis. Statistical heterogeneity between trials was detected by the Chi-squared and $I$-square $\left(I^{2}\right)$ tests. In the event of significant heterogeneity $\left(P<0.1\right.$ and $\left.I^{2}>50 \%\right)$, the random-effect model was used for analysis; in other cases, a fixed-effect model was used. Whole effects with $P$-value $<0.05$ indicated statistical significance between experimental and control groups. SMD value approaching $0(P>0.05)$ was judged to have no statistical significance. In contrast, $\mathrm{SMD}$ value deviating from 0 $(P<0.05)$ was regarded as a "significant finding". Subgroup analyses were conducted to explore heterogeneity among studies with respect to underlying disease and form of intervention (turmeric or curcumin) used. As only a single study had reported efficacy measures disaggregated by gender [27], subgroup analyses by gender could not be undertaken. To assess publication bias, funnel plots and Begg's test were conducted initially.
However, as fewer than 10 studies were selected in each meta-analysis, the funnel plots and Begg's test could not be conducted.

\section{Results}

\section{Summary of included studies}

Of the 1566 articles retrieved on initial literature search, a detailed evaluation of 11 full-text articles resulted in the elimination of 2 studies [33, 34], due to the use of the same patient groups, and another 2 studies [35, 36], owing to incomplete data (Fig. 1). Consequently, 7 randomized controlled trials (RCTs), including a total of 649 subjects, met the inclusion criteria and were selected for a qualitative analysis.

Basic characteristics of the included RCTs are summarized in Table 1, and the pre- and post-intervention serum lipid parameters are presented in Additional file 1: Table S1. Of the 7 eligible studies, only studies that enrolled patients with T2DM $[15,24,25,28]$ or $\operatorname{MetS}[23,26,27]$ were included. All studies were conducted in a doubleblind manner with the exception of the trials by Selvi et al. [25] and Usharani et al. [15]. Two of the studies were performed in Iran $[23,24]$ and $2[15,25]$ were conducted in India. The remaining studies were conducted in Pakistan [26], Taiwan [27], and Thailand [28], respectively. The duration of these studies ranged from 4 weeks to 6 months. Specified outcomes were reported from all studies, with the exception of the study by Chuengsamarn et al. [28], for which only serum TG levels were reported. The form of intervention and serum lipid parameters of studies at baseline and after intervention differed.

\section{Data quality}

The risk of bias in the individual studies is shown in Additional file 2: Table S2. Overall, these selected studies varied in terms of quality: of the 7 RCTs, 4 were classified as high quality [24, 26-28] and 3 were judged to be of moderate quality $[15,23,25]$. Four studies used appropriate randomization methods, such as a random number table $[25,27]$ or a computer-generated list of random numbers [24, 28]. Allocation concealment was only used in 4 studies [23, 25, 26, 28]. Five trials used double-blinding of patients and practitioners [23, 24, 26-28]. All studies reported dropout rates and specific reasons for dropout with the exception of the trial by Chuengsamarn et al. [28].

\section{Meta-analysis}

Pooled data from 6 trials [15, 23-27] ( $n=218$ both cases and controls) showed significant efficacy of the study drug in reducing serum LDL-C levels; no significant heterogeneity was observed between these six trials $\left(P<0.0001, I^{2}=42.10 \%\right.$, Fig. 2$)$. Similarly, turmeric and curcumin therapy did not exhibit a favorable effect on serum HDL-C levels ( $P=0.370, I^{2}=0.00 \%$, Fig. 3$)$. Meta- 


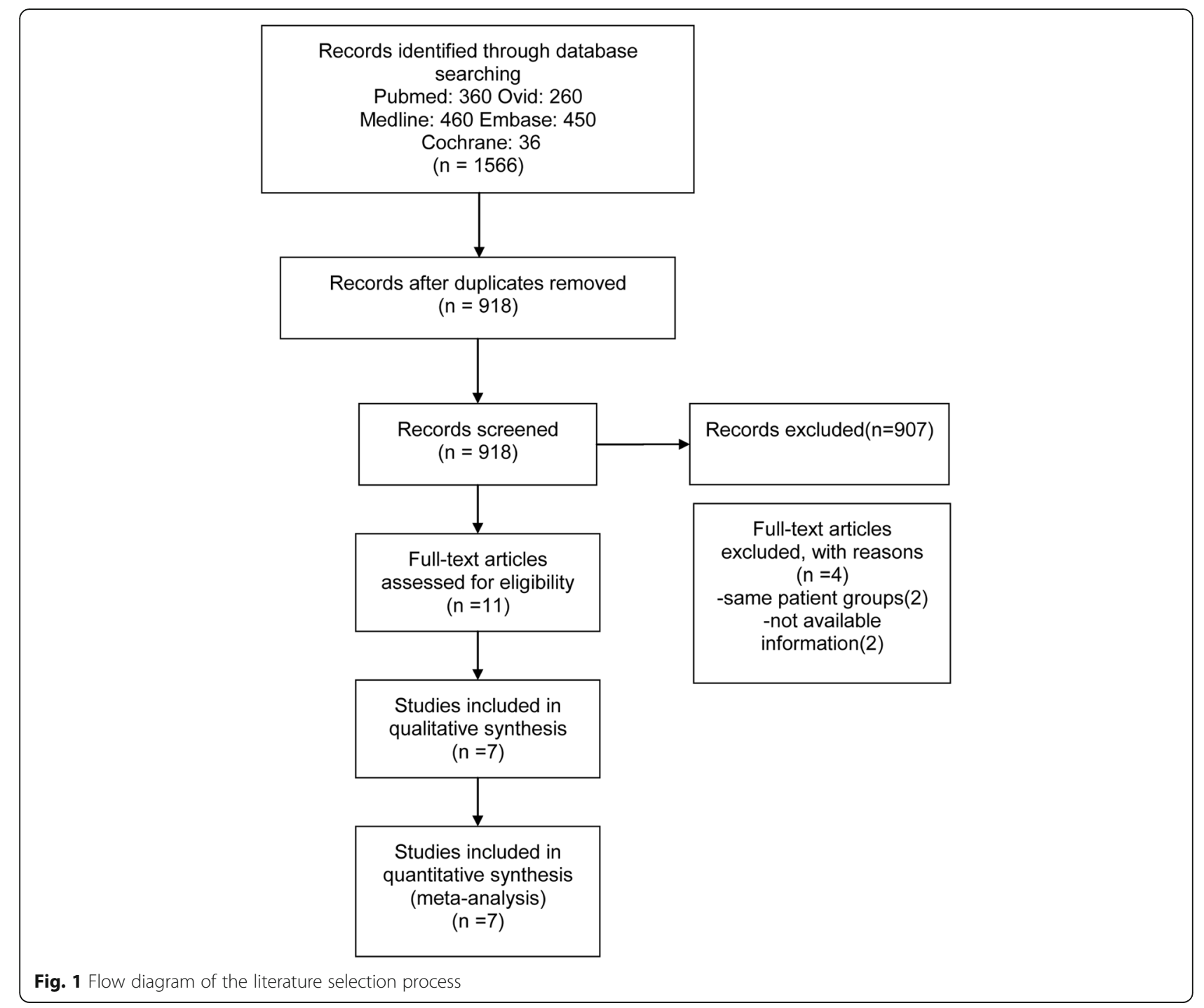

analysis of data from 7 studies [15, 23-28] indicated an obvious benefit of experimental treatment $(n=325)$ in reducing serum TG levels, compared to that with control treatment $(n=324)(\mathrm{SMD}=-0.214,95 \%$ CI: -0.369 to $-0.059, P=0.007, I^{2}=24.5 \%$, Fig. 4). Although a randomeffect model was used, pooled analysis of data from 6 studies [15, 23-27] showed no significant between-group differences in terms of plasma TC concentrations $(P=0.054)$, ostensibly owing to the significant heterogeneity among these studies $\left(I^{2}=73.8 \%, n=218\right.$ for both experiment and control groups, Fig. 5).

\section{Subgroup analyses}

To assess sources of potential bias, we conducted subgroup analyses by underlying diseases and forms of intervention-namely, studies in patients with hyperglycemia (prediabetes and T2DM) and MetS (Table 2). Two studies [23, 27] which included patients with MetS revealed significant differences $\left(P<0.0001, I^{2}=0.00 \%\right)$ with respect to serum TC levels between the experimental $(n=67)$ and placebo $(n=69)$ groups. However, pooled data from 4 trials [15, 24-26] showed no significant differences in this respect $\left(P=0.612, I^{2}=0.00 \%\right)$ among patients with hyperglycemia, between the experimental $(n=151)$ and placebo $(n=149)$ groups. With regard to form of intervention, analysis of pooled data from 2 studies [25, 26] comprising 186 subjects showed no favorable effect of turmeric powder therapy on serum TC levels $[-0.000(-0.288$ to 0.288$), P=0.999$, $\left.I^{2}=17.1 \%\right]$. However, 2 studies $[15,27]$ showed significant benefits of turmeric extract $\left(P=0.004, I^{2}=31.1 \%\right)$ in the experimental group $(n=53)$ as compared to that in the control group $(n=50)$.

\section{Side effects of turmeric and curcumin}

Five RCTs reported adverse effects in both the control and experimental groups. Rahmani et al. [23] reported 
Table 1 Characteristics of studies included in the meta-analysis

\begin{tabular}{|c|c|c|c|c|c|c|c|c|c|}
\hline Study & Region & Type & Duration & Inclusion criteria & & Intervention & $N$ & Age & Male \\
\hline \multicolumn{10}{|c|}{ Metabolic syndrome } \\
\hline \multirow[t]{2}{*}{$\begin{array}{l}\text { Rahmani et al. } \\
2016 \text { [23] }\end{array}$} & \multirow[t]{2}{*}{ Iran } & \multirow[t]{2}{*}{ RCT double-blind } & \multirow[t]{2}{*}{8 weeks } & \multirow[t]{2}{*}{$\begin{array}{l}\text { Patients with } \\
\text { symptoms of MetS } \\
\text { (NCEP-ATP III) and } \\
\text { diagnosis of NAFLD }\end{array}$} & Case & $\begin{array}{l}\text { Amorphous dispersion } \\
\text { Curcumin (amorphous } \\
\text { dispersion preparation, } \\
\text { equivalent to } 70 \mathrm{mg} / \mathrm{d} \\
\text { curcuminoids) }\end{array}$ & 37 & $46.37 \pm 11.57$ & 19 \\
\hline & & & & & Control & Placebo & 40 & $48.95 \pm 9.78$ & 19 \\
\hline \multirow[t]{2}{*}{$\begin{array}{l}\text { Amin et al. } \\
2015 \text { [26] }\end{array}$} & \multirow[t]{2}{*}{ Pakistan } & \multirow[t]{2}{*}{ RCT double-blind } & \multirow[t]{2}{*}{8 weeks } & \multirow{2}{*}{$\begin{array}{l}\text { Patients with } \geq 3 \\
\text { features of MetS, } \\
\text { prediabetes, } \\
\text { dyslipidemia and } \\
\text { prehypertension }\end{array}$} & Case & $\begin{array}{l}\text { Turmeric powder } \\
2.4 \mathrm{~g} / \mathrm{d}\end{array}$ & 63 & $42.40 \pm 13.70$ & NA \\
\hline & & & & & Control & Placebo & 63 & $41.57 \pm 12.80$ & NA \\
\hline \multirow[t]{2}{*}{$\begin{array}{l}\text { Yang et al. } \\
2014 \text { [27] }\end{array}$} & \multirow[t]{2}{*}{ Taiwan } & \multirow[t]{2}{*}{ RCT double-blind } & \multirow[t]{2}{*}{12 weeks } & \multirow[t]{2}{*}{$\begin{array}{l}\text { Patients with } \\
\text { diagnosis of MetS } \\
\text { (NCEP-ATP III) }\end{array}$} & Case & $\begin{array}{l}\text { Turmeric extract } \\
\text { (equivalent to } 1890 \\
\text { mg/d curcuminods) }\end{array}$ & 30 & $59.03 \pm 10.10$ & 12 \\
\hline & & & & & Control & Placebo & 29 & $59.61 \pm 14.09$ & 17 \\
\hline \multicolumn{10}{|c|}{ Type 2 diabetes mellitus } \\
\hline \multirow[t]{2}{*}{$\begin{array}{l}\text { Rahimi et al. } \\
2016 \text { [24] }\end{array}$} & \multirow[t]{2}{*}{ Iran } & \multirow[t]{2}{*}{ RCT double-blind } & \multirow[t]{2}{*}{3 months } & \multirow[t]{2}{*}{$\begin{array}{l}\text { Type } 2 \text { Diabetic } \\
\text { patients }\end{array}$} & Case & $\begin{array}{l}\text { Curcumin (nano- } \\
\text { micelle } 80 \text { mg/day) }\end{array}$ & 35 & $56.34 \pm 11.17$ & 17 \\
\hline & & & & & Control & placebo & 35 & $60.95 \pm 10.77$ & 14 \\
\hline \multirow[t]{2}{*}{$\begin{array}{l}\text { Selvi et al. } \\
2015 \text { [25] }\end{array}$} & \multirow[t]{2}{*}{ India } & \multirow[t]{2}{*}{$\mathrm{RCT}$} & \multirow[t]{2}{*}{4 weeks } & \multirow[t]{2}{*}{$\begin{array}{l}\text { Type } 2 \text { diabetic } \\
\text { patients }\end{array}$} & Case & $\begin{array}{l}\text { Turmeric powder } \\
2 \mathrm{~g} / \text { day +Metformin }\end{array}$ & 30 & $47.00 \pm 7.17$ & 30 \\
\hline & & & & & Control & Metformin (1 g/day) & 30 & $46.80 \pm 6.10$ & 30 \\
\hline \multirow[t]{2}{*}{$\begin{array}{l}\text { Chuengsamarn } \\
\text { et al. } 2014 \text { [28] }\end{array}$} & \multirow[t]{2}{*}{ Thailand } & \multirow[t]{2}{*}{ RCT double-blind } & \multirow[t]{2}{*}{6 months } & \multirow[t]{2}{*}{$\begin{array}{l}\text { Type } 2 \text { diabetic } \\
\text { patients }\end{array}$} & Case & $\begin{array}{l}\text { Turmeric extract } \\
\text { (equivalent to } 1500 \\
\text { mg/d curcuminods) }\end{array}$ & 107 & $59.16 \pm 11.04$ & 50 \\
\hline & & & & & Control & Placebo & 106 & $59.58 \pm 10.71$ & 47 \\
\hline \multirow[t]{2}{*}{$\begin{array}{l}\text { Usharani et al. } \\
2008 \text { [15] }\end{array}$} & \multirow[t]{2}{*}{ India } & \multirow[t]{2}{*}{$\mathrm{RCT}$} & \multirow[t]{2}{*}{8 weeks } & $\begin{array}{l}\text { Type } 2 \text { diabetic } \\
\text { patients }\end{array}$ & Case & $\begin{array}{l}\text { Turmeric extract } \\
\text { (equivalent to } 600 \\
\text { mg/day curcuminoids) }\end{array}$ & 23 & $55.52 \pm 10.76$ & 12 \\
\hline & & & & & Control & Placebo & 21 & $49.75 \pm 8.18$ & 11 \\
\hline
\end{tabular}

MetS metabolic syndrome, NAFLD nonalcoholic fatty liver disease, NCEP-ATP III National Cholesterol Education Program Adult Treatment Panel III, a diagnostic guideline of MetS, RCT randomized controlled trial, NA not available

Values are expressed as mean \pm SD

that 2 patients experienced simultaneous abdominal pain and nausea, whereas another patient suffered from abdominal pain. Amin et al. [26] reported adverse events such as nausea and dyspepsia, but failed to report the exact number of subjects who experienced these events. Chuengsamarn et al. [28] reported side effects of curcumin in 4 patients: constipation in 2, hot flashes in 1 , and nausea in 1 patient. Moreover, 4 patients experienced side effects in the placebo group: vertigo and itching, constipation, and hot flashes in 1 patient each. Selvi et al. [25] reported mild diarrhea in 2 subjects. None of the remaining studies reported any adverse reactions of turmeric and curcumin therapy. No serious adverse reaction induced by turmeric and curcumin was reported in any of the studies included in this meta-analysis.

\section{Discussion}

The epidemic of obesity has contributed to a growing burden of CVD risk factors such as T2DM and MetS [37] (defined as the presence of at least 3 out of the 4 criteria: central obesity, increased blood pressure, high blood sugar levels, and dyslipidemia [38]). Dyslipidemia is a wellestablished modifiable cardiovascular risk factor. All of the currently available antilipemic therapies have their own inherent shortcomings and disadvantages. Therefore, natural treatments have been investigated as potential therapies for lowering blood lipid levels.

This systematic review of 7 randomized trials of turmeric and curcumin in patients at risk of CVD identified evidence of their beneficial effects on serum TG and LDL-C levels, although no significant difference was found with respect to serum HDL levels. Despite the use of random-effects model to compensate for heterogeneity, no statistically significant benefit was observed with regard to TC $(P=0.054)$. When the analysis was restricted to more homogenous studies based on underlying disease in subjects (hyperglycemia and MetS), a beneficial effect of turmeric and curcumin on serum TC 


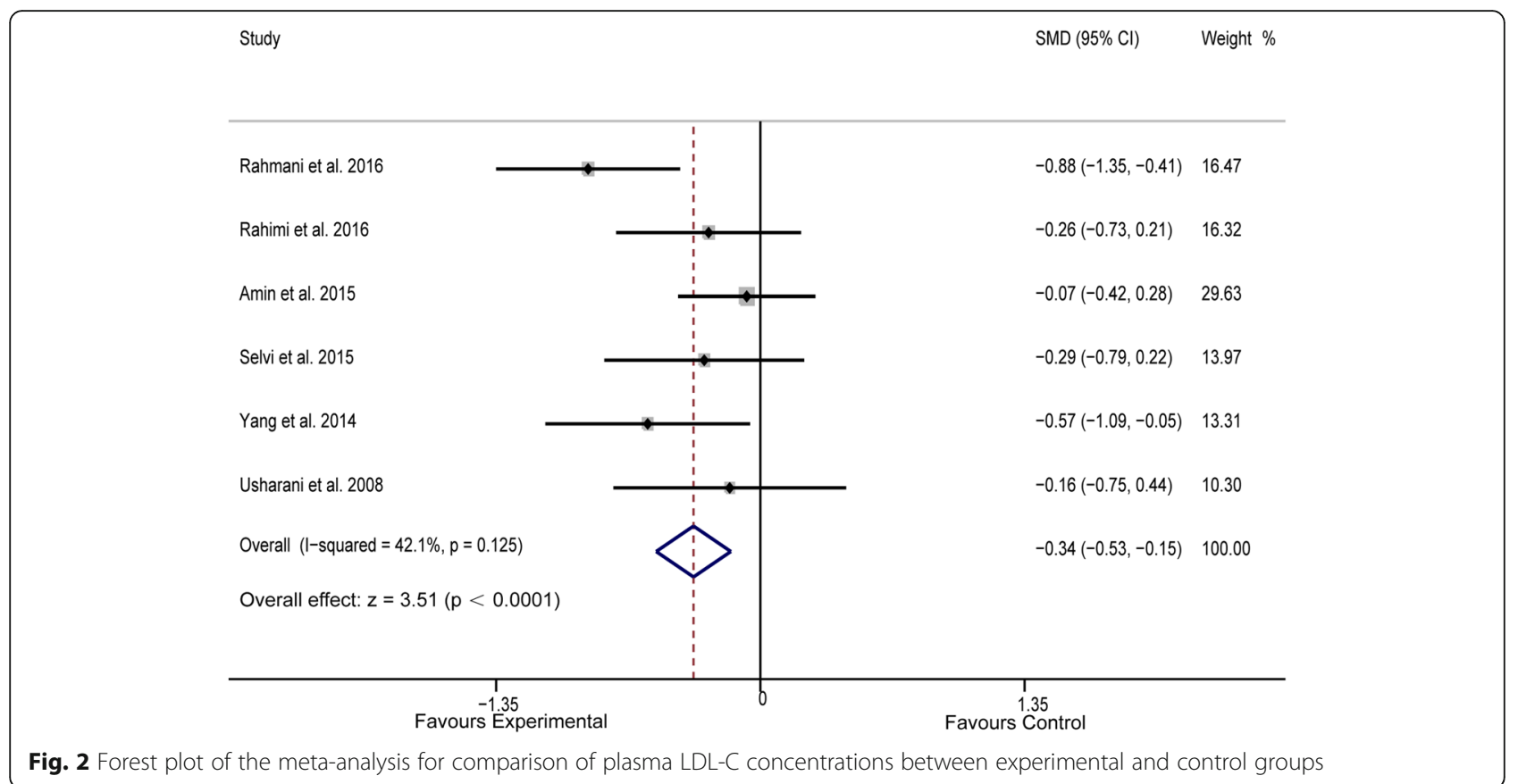

levels was observed in subjects with MetS; however, in subjects with hyperglycemia, this beneficial effect on serum TC levels was not observed. It seems that the natural form (turmeric) and curcumin have more positive effects on patients suffering from MetS. With regard to the forms of intervention, turmeric extract may have a greater beneficial effect on serum TC levels, as compared to that of turmeric in its natural form. However, owing to the limited number of studies, definitive conclusions may not be drawn in this respect. Furthermore, larger scale trials are required among patients with MetS to explore the effect of turmeric extract, even in novel forms, in lowering plasma TC concentrations.

Sahebkar conducted a meta-analysis of 5 RCTs to assess the effects of curcumin on blood lipid levels and found no significant improvements in the lipid profile in

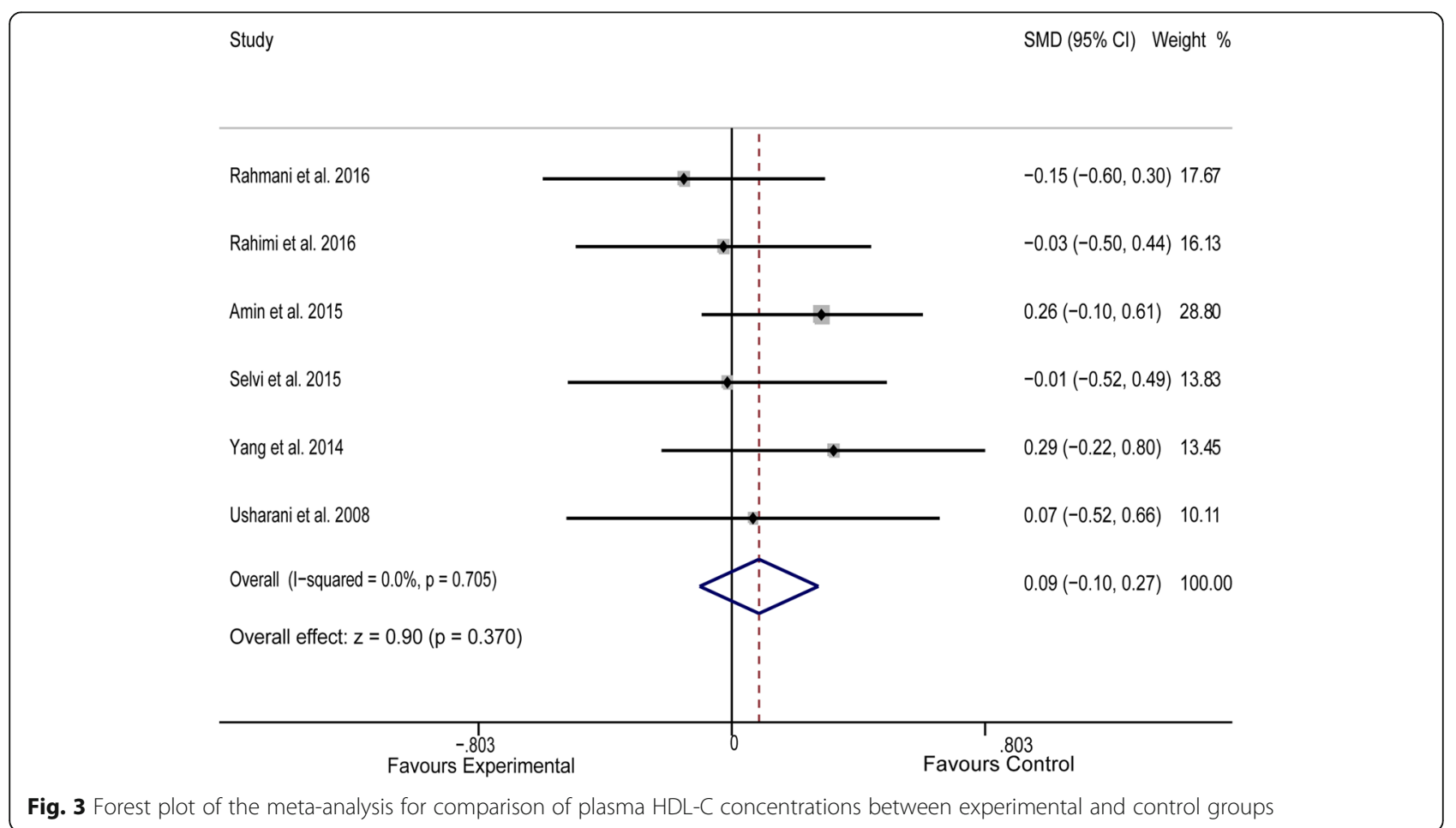




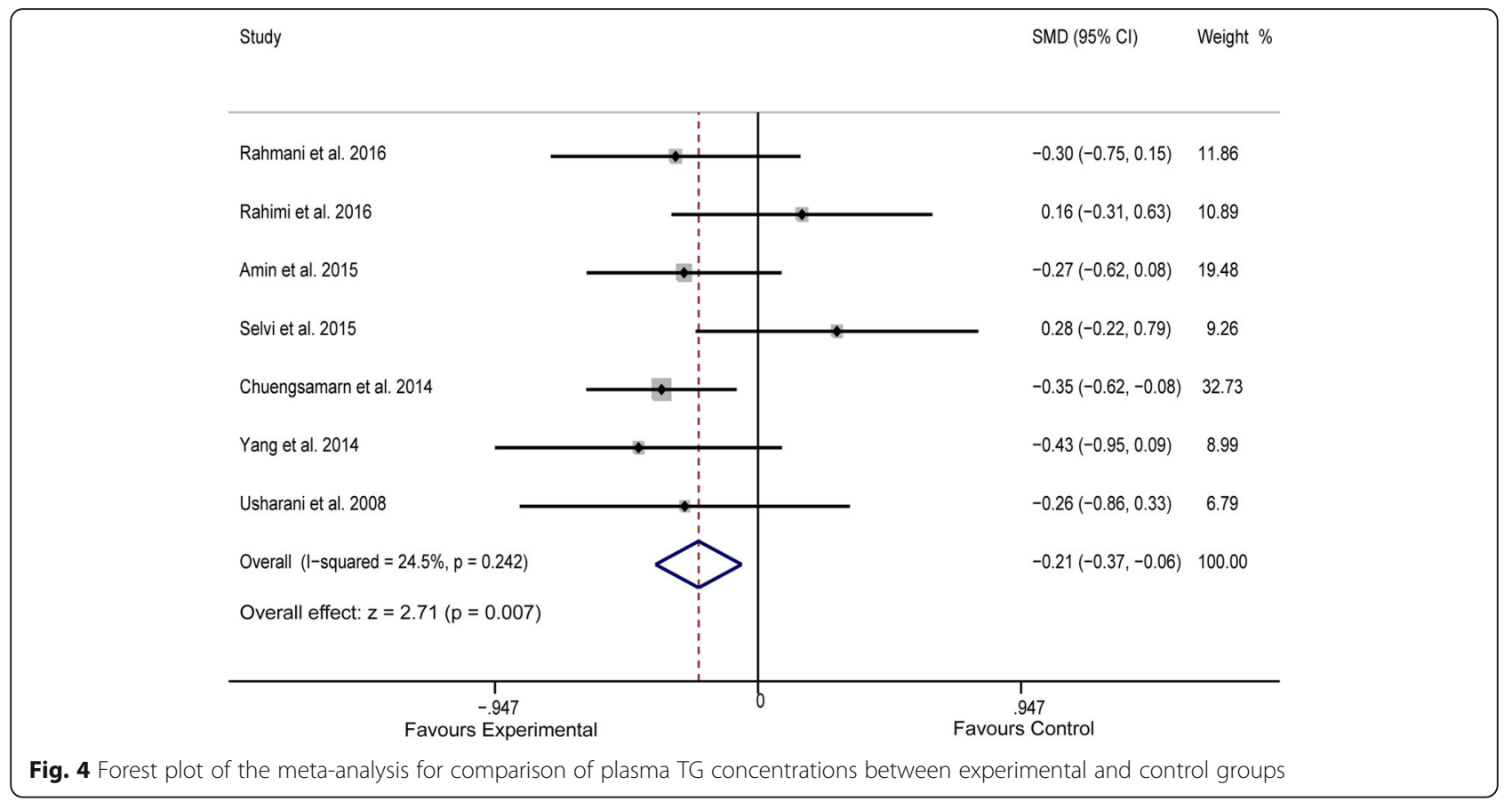

any aspect [22]. Several explanations could be tendered to explain why the results of their study were contrary to those of the present study. Firstly, both parallel and crossover randomized trials were selected, and these may have adversely influenced the final results.

Secondly, most of the selected studies were conducted with unformulated curcumin, which is considered to have low bioavailability. Curcumin has poor bioavailability owing to its poor absorption, fast metabolism, and rapid elimination from the body. Some attempts have been made to overcome these deficits, including the use of a piperine (black pepper) adjuvant, liposomal curcumin, nanoparticles, phospholipid complexes, and an amorphous form $[39,40]$. Therefore, a hypothesis could be proposed that these novel dosage forms of curcumin may achieve greater clinical effects. To verify this theory, forest plots were conducted initially. Nevertheless, in our present review, only two trials [23, 24] used novel

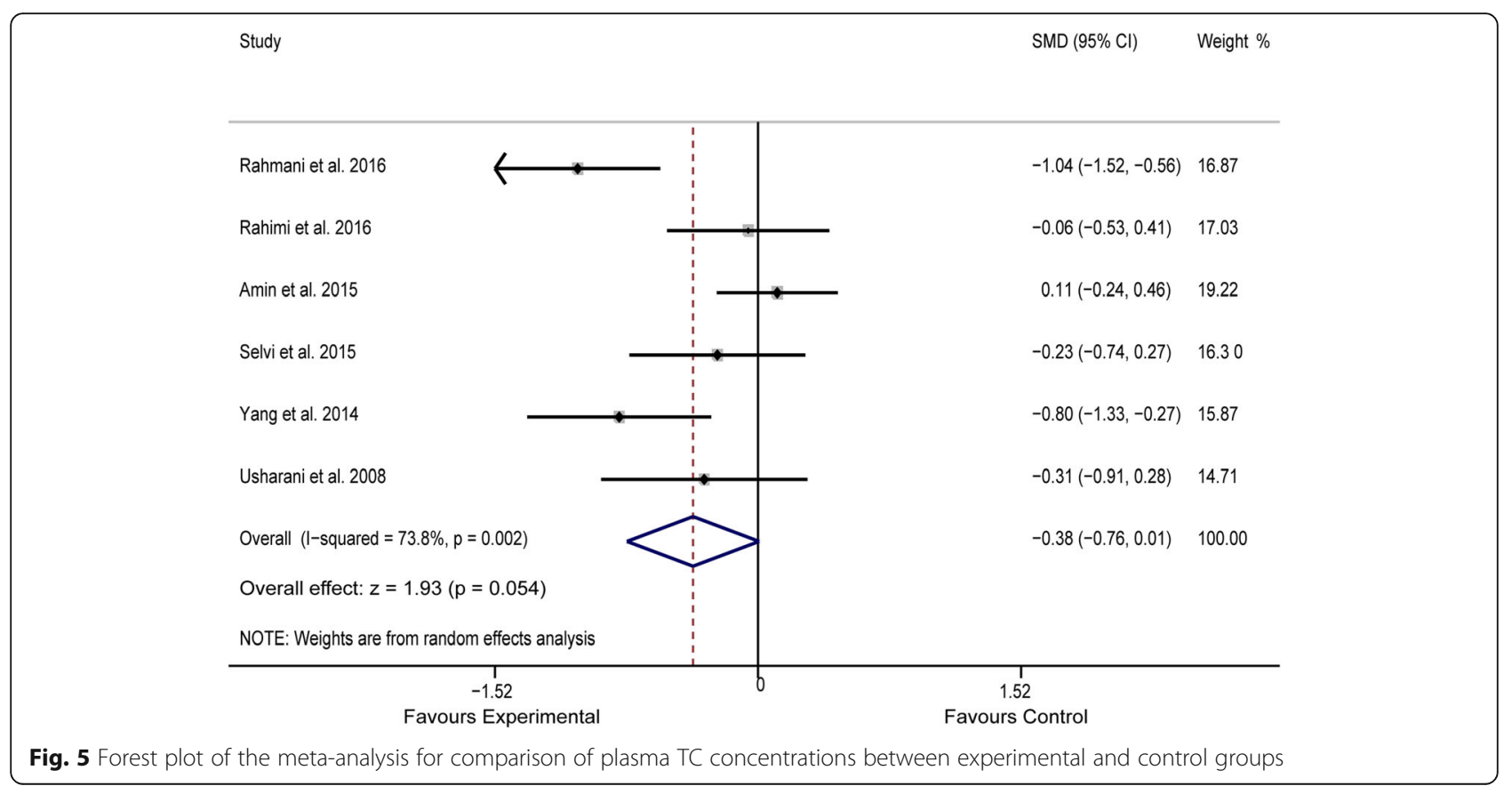


Table 2 Subgroup analysis of serum total cholesterol (TC) levels

\begin{tabular}{|c|c|c|c|c|c|c|c|c|c|c|}
\hline \multicolumn{3}{|l|}{ Outcome of interest } & \multirow{2}{*}{$\frac{\text { No. of }}{\text { study }}$} & \multirow{2}{*}{$\frac{\text { No. of }}{\text { patients }}$} & \multirow{2}{*}{$\frac{\text { Effects }}{\text { model }}$} & \multirow[t]{2}{*}{ SMD } & \multirow[t]{2}{*}{$95 \% \mathrm{Cl}$} & \multicolumn{2}{|c|}{ Heterogeneity } & \multirow[t]{3}{*}{$P$-value } \\
\hline & & & & & & & & $P$ value & $P^{2}(\%)$ & \\
\hline & & & arms & & & & & & & \\
\hline \multirow[t]{5}{*}{ TC } & & & 6 & 436 & Random & -0.375 & -0.757 to 0.006 & 0.002 & 73.80 & 0.054 \\
\hline & Form of intervention & Turmeric powder & 2 & 186 & Fixed & -0.000 & -0.288 to 0.288 & 0.272 & 17.10 & 0.999 \\
\hline & & Turmeric extract & 2 & 103 & Fixed & -0.584 & -0.980 to -0.188 & 0.228 & 31.10 & $0.004^{*}$ \\
\hline & Type of disease & MetS & 2 & 136 & Fixed & -0.934 & -1.289 to -0.579 & 0.510 & 0.000 & $<0.0001^{* *}$ \\
\hline & & Hyperglycemia & 4 & 300 & Fixed & -0.059 & -0.285 to 0.168 & 0.561 & 0.000 & 0.612 \\
\hline
\end{tabular}

MetS metabolic syndrome

${ }^{*} p<0.05$

** $p<0.001$

${ }^{* * *} p<0.0001$

forms, and the preparations used in these studies were dissimilar (amorphous forms were used in one study [23] and nanoparticles were used in the other [24]). Therefore, further research on newer dosage forms of curcumin is required to confirm the hypothesis.

Finally, differences with respect to underlying diseases in the study population may also explain this discrepancy. The authors included healthy participants and patients with various chronic diseases (i.e., coronary artery disease, diabetes, Alzheimer's disease, or obesity), which may have resulted in different outcomes. Even on performing a subgroup analysis in patients with high cardiovascular risk (acute coronary syndrome, T2DM, or concomitant dyslipidemia and obesity), no significant difference could be identified because coronary artery disease is an end event rather than a risk factor for CVD.

The article by Mohammadi et al. [19] was not included in the meta-analyses because its design as a randomized crossover trial did not fulfill the inclusion criteria. However, the study investigated the hypothesis that curcuminoids (1 g/day for 30 days) lead to a significant reduction in serum triglyceride concentrations in obese individuals. Unlike this study, in a trial among patients with coronary artery disease, although curcumin supplementation decreased serum levels of TC, LDL-C, and TG, there was no obvious difference when compared to placebo [41], possibly due to the small size of the study. Subsequently, a study by Soare et al. found that $900 \mathrm{mg}$ of curcumin did not influence plasma lipid levels in non-obese relatively healthy individuals [42]. Therefore, we tentatively propose that the antilipemic effect of curcumin is evident only in patients who are at a higher risk of cardiovascular morbidity, such as those with MetS, T2DM, and obesity.

Some molecular mechanisms could potentially explain these results. Insulin resistance (IR) is the basic underlying pathology in both T2DM and MetS. Neerati et al. reported that curcumin could counter IR [43]. Through amelioration of metabolic derangement and potential binding of curcumin with peroxisome proliferator-activated receptor gamma
(PPAR- $\gamma$ ) as agonist, curcumin could play a preventive role in diet-induced insulin resistance [44]. Moreover, curcumin was shown to increase activation of PPAR- $\gamma$ [45], which suppressed expression of the LDL-C receptor gene, and could thereby reduce plasma LDL-C concentrations [46]. Because it interacts with multiple targets, including peroxisome proliferator-activated receptor alpha (PPAR- $\alpha$ ), PPAR$\gamma$, cholesteryl ester transfer protein (CETP), and lipoprotein lipase, curcumin could probably play a role in reduction of triglyceride levels [47-49]. Furthermore, curcumin is expected to affect both synthesis and catabolism of triglyceride-rich lipoproteins [47-49]. Thus, curcumin supplementation may lower plasma triglycerides and cholesterol concentrations by mitigating the expressions of lipogenic genes [48-50]. Additionally, the lipid-lowering effect of turmeric and curcumin is related to statins. Panahi et al. found that curcumin affected all pathways of cholesterol metabolism that are affected by statin therapy; it also reduced the effective doses of statins, which helped reduce the incidence of serious adverse reactions [51]. Furthermore, curcumin might serve as a valuable adjunct to statin therapy in patients with disordered lipid metabolism [51].

In our meta-analysis, we found that consumption of turmeric and curcumin was safe and well-tolerated in general. This finding is consistent with those of previous studies in human subjects [10], and dosages as high as $8000 \mathrm{mg} /$ day have been shown to be well-tolerated with no apparent toxicity [52].

Several potential limitations of this review need mention. First, the most important limitation may pertain to the interpretability of outcomes. Second, this review did not include unpublished studies or studies published in the "grey literature". Third, all subjects in the included studies were Asians. Lastly, some data were obtained indirectly, and those could have affected the accuracy of both the overall effects and the results of subgroup analyses.

\section{Conclusions}

Subjects who received turmeric and curcumin experienced a natural cardioprotective effect, with lowering of 
serum LDL-C and TG levels, as compared to subjects who did not. The efficacy of turmeric and curcumin on serum TC levels remains inconclusive, despite their superior efficacy observed in patients with MetS. A greater effect of turmeric extract in reducing serum TC levels may be observed in patients who are at risk of CVD; however, this finding needs to be confirmed in future studies. No significant change in serum HDL levels was observed.

Because curcumin's poor bioavailability limits its absorption from dietary sources, novel formulations with enhanced bioavailability are probably required to control dyslipidemia more effectively.

Due to uncertainties related to dosage form, dose and medication frequency, it is premature to recommend the use of turmeric or curcumin in clinical settings. Nonetheless, the analysis does provide a synthesis of the currently available evidence and supports larger scale clinical trials of curcumin.

\section{Additional files}

Additional file 1: Table S1. Serum lipid parameters in studies from before and after intervention. (DOC $64 \mathrm{~kb}$ )

Additional file 2: Table S2. Quality of studies assessed by the Cochrane guidelines. (DOC $37 \mathrm{~kb}$ )

\section{Abbreviations}

CETP: Cholesteryl ester transfer protein; Cl: Confidence interval; CVD: Cardiovascular disease; HDL-C: High-density lipoprotein cholesterol; IR: Insulin resistance; LDL-C: Low-density lipoprotein cholesterol; MeSH: Medical Subject Heading; MetS: Metabolic syndrome; PPARa: Peroxisome proliferator-activated receptor alpha; PPAR- $\gamma$ : Peroxisome proliferator-activated receptor gamma; RCTs: Randomized controlled trials; SD: Standard deviation; SE: Standard error; SMD: Standardized mean difference; T2DM: Type 2 diabetes mellitus; TC: Total cholesterol; TG: Triglycerides

\section{Acknowledgements}

Not applicable.

\section{Funding}

This research was supported by the Natural Science Foundation of China (grant no. 81171560), the "Par-Eu Scholars Program" of Chongqing City and the National Science and Technology Major Project of China (grant no. 2012ZX10002007001). The funding bodies had no role in study design, data collection and analysis, decision to publish or preparation of the manuscript.

\section{Availability of data and materials}

The tables and figures supporting the conclusions of this article are included within the article. There are also two supplementary tables online.

\section{Authors' contributions}

$\mathrm{SQ}, \mathrm{HR}$ and $\mathrm{HDH}$ contributed to the conception and design of the study. SQ $\mathrm{LFH}$ and $\mathrm{HDH}$ conducted the literature search and data extraction. $\mathrm{LFH}$ and SQ performed the statistical analyses. SQ, LFH, JJG, SSS, JH and HDH drafted the manuscript. HR and HDH supervised the study. All authors gave final approval.

\section{Ethics approval and consent to participate}

Not applicable.

\section{Consent for publication}

Not applicable.

\section{Competing interests}

The authors declare that they have no competing interests.

\section{Publisher's Note}

Springer Nature remains neutral with regard to jurisdictional claims in published maps and institutional affiliations.

Received: 10 March 2017 Accepted: 5 October 2017

Published online: 11 October 2017

\section{References}

1. World Health Organization: The Top 10 Causes of Death. http://www. who.int/mediacentre/factsheets/fs310/en/index4.html (2014). Accessed 20 Nov 2016.

2. Srikanth S, Deedwania P. Management of Dyslipidemia in patients with hypertension, diabetes, and metabolic syndrome. Curr Hypertens Rep. 2016;18:76

3. Musunuru K. Atherogenic dyslipidemia: cardiovascular risk and dietary intervention. Lipids. 2010;45:907-14.

4. Padala S, Thompson PD. Statins as a possible cause of inflammatory and necrotizing myopathies. Atherosclerosis. 2012;222:15-21.

5. Chalasani N. Statins and hepatotoxicity: focus on patients with fatty liver. Hepatology. 2005:41:690-5

6. Sniderman $A D$. Is there value in liver function test and creatine phosphokinase monitoring with statin use? Am J Cardiol. 2004;94:30F-4F.

7. Law M, Rudnicka AR. Statin safety: a systematic review. Am J Cardiol. 2006; 97:52C-60C

8. Goel A, Kunnumakkara AB, Aggarwal BB. Curcumin as "Curecumin": from kitchen to clinic. Biochem Pharmacol. 2008;75:787-809.

9. Sahebkar A. Why it is necessary to translate curcumin into clinical practice for the prevention and treatment of metabolic syndrome? Biofactors. 2013; 39:197-208

10. Kunnumakkara AB, Bordoloi D, Padmavathi G, Monisha J, Roy NK, Prasad S, Aggarwal BB. Curcumin, the golden nutraceutical: multitargeting for multiple chronic diseases. Br J Pharmacol. 2016; 10.1111/bph.1362.

11. Pulido-Moran M, Moreno-Fernandez J, Ramirez-Tortosa C, Ramirez-Tortosa M. Curcumin and health. Molecules. 2016;21:264

12. Maithilikarpagaselvi N, Sridhar MG, Swaminathan RP, Sripradha R, Badhe B. Curcumin inhibits hyperlipidemia and hepatic fat accumulation in highfructose-fed male Wistar rats. Pharm Biol. 2016;54:2857-63.

13. Li ZY, Ding LL, Li JM, Xu BL, Yang L, Bi KS, Wang ZT. (1)H-NMR and MS based metabolomics study of the intervention effect of curcumin on hyperlipidemia mice induced by high-fat diet. PLoS One. 2015;10:e0120950.

14. Shin SK, Ha TY, McGregor RA, Choi MS. Long-term curcumin administration protects against atherosclerosis via hepatic regulation of lipoprotein cholesterol metabolism. Mol Nutr Food Res. 2011:55:1829-40.

15. Usharani P, Mateen AA, Naidu MU, Raju YS, Chandra N. Effect of NCB-02, atorvastatin and placebo on endothelial function, oxidative stress and inflammatory markers in patients with type 2 diabetes mellitus: a randomized, parallel-group, placebo-controlled, 8-week study. Drugs R D. 2008;9:243-50

16. Soni KB, Kuttan R. Effect of oral curcumin administration on serum peroxides and cholesterol levels in human volunteers. Indian J Physiol Pharmacol. 1992;36:273-5.

17. Ramirez-Bosca A, Soler A, Carrion MA, Diaz-Alperi J, Bernd A, Quintanilla C, Quintanilla Almagro E, Miquel J. An hydroalcoholic extract of curcuma longa lowers the apo B/apo a ratio. Implications for atherogenesis prevention. Mech Ageing Dev. 2000;119:41-7.

18. Pungcharoenkul $K$, Thongnopnua $P$. Effect of different curcuminoid supplement dosages on total in vivo antioxidant capacity and cholesterol levels of healthy human subjects. Phytother Res. 2011;25:1721-6.

19. Mohammadi A, Sahebkar A, Iranshahi M, Amini M, Khojasteh R, GhayourMobarhan M, Ferns GA. Effects of supplementation with curcuminoids on dyslipidemia in obese patients: a randomized crossover trial. Phytother Res. 2013;27:374-9.

20. Baum L, Cheung SK, Mok VC, Lam LC, Leung VP, Hui E, Ng CC, Chow M, Ho PC, Lam S, et al. Curcumin effects on blood lipid profile in a 6-month human study. Pharmacol Res. 2007:56:509-14.

21. Alwi I, Santoso T, Suyono S, Sutrisna B, Suyatna FD, Kresno SB, Ernie S. The effect of curcumin on lipid level in patients with acute coronary syndrome. Acta Med Indones. 2008;40:201-10. 
22. Sahebkar A. A systematic review and meta-analysis of randomized controlled trials investigating the effects of curcumin on blood lipid levels. Clin Nutr. 2014;33:406-14.

23. Rahmani S, Asgary S, Askari G, Keshvari M, Hatamipour M, Feizi A, Sahebkar A. Treatment of non-alcoholic fatty liver disease with Curcumin: a randomized placebo-controlled trial. Phytother Res. 2016:30:1540-8.

24. Rahimi HR, Mohammadpour AH, Dastani M, Jaafari MR, Abnous K, Ghayour Mobarhan M, Kazemi OR. The effect of nano-curcumin on $\mathrm{HbA1c}$, fasting blood glucose, and lipid profile in diabetic subjects: a randomized clinical trial. Avicenna J Phytomed. 2016;6:567-77.

25. Maithili Karpaga Selvi N, Sridhar MG, Swaminathan RP, Sripradha R. Efficacy of turmeric as adjuvant therapy in type 2 diabetic patients. Indian J Clin Biochem. 2015;30:180-6

26. Amin F, Islam N, Anila N, Gilani AH. Clinical efficacy of the co-administration of turmeric and black seeds (Kalongi) in metabolic syndrome - a double blind randomized controlled trial - TAK-MetS trial. Complement Ther Med. 2015:23:165-74

27. Yang YS, Su YF, Yang HW, Lee YH, Chou Jl, Ueng KC. Lipid-lowering effects of curcumin in patients with metabolic syndrome: a randomized, doubleblind, placebo-controlled trial. Phytother Res. 2014;28:1770-7.

28. Chuengsamarn S, Rattanamongkolgul S, Phonrat B, Tungtrongchitr R, Jirawatnotai S. Reduction of atherogenic risk in patients with type 2 diabetes by curcuminoid extract: a randomized controlled trial. J Nutr Biochem. 2014;25:144-50

29. Daily JW, Yang M, Park S. Efficacy of turmeric extracts and Curcumin for alleviating the symptoms of joint arthritis: a systematic review and metaanalysis of randomized clinical trials. J Med Food. 2016;19:717-29.

30. Higgins JP, Altman DG, Gotzsche PC, Juni P, Moher D, Oxman AD, Savovic J, Schulz KF, Weeks L, Sterne JA, et al. The Cochrane Collaboration's tool for assessing risk of bias in randomised trials. BMJ. 2011;343:d5928.

31. Higgins JPT, Green S, editors. Cochrane handbook for systematic reviews of interventions version 5.0.2. The Cochrane Collaboration; 2009.

32. Hozo SP, Djulbegovic B, Hozo I. Estimating the mean and variance from the median, range, and the size of a sample. BMC Med Res Methodol. 2005;5:13.

33. Panahi Y, Khalili N, Hosseini MS, Abbasinazari M, Sahebkar A. Lipidmodifying effects of adjunctive therapy with curcuminoids-piperine combination in patients with metabolic syndrome: results of a randomized controlled trial. Complement Ther Med. 2014;22:851-7.

34. Sahebkar A, Mohammadi A, Atabati A, Rahiman S, Tavallaie S, Iranshahi M, Akhlaghi S, Ferns GA, Ghayour-Mobarhan M. Curcuminoids modulate pro-oxidant-antioxidant balance but not the immune response to heat shock protein 27 and oxidized LDL in obese individuals. Phytother Res. 2013;27:1883-8

35. Panahi Y, Hosseini MS, Khalili N, Naimi E, Soflaei SS, Majeed M, Sahebkar A. Effects of supplementation with curcumin on serum adipokine concentrations: a randomized controlled trial. Nutrition. 2016;32:1116-22.

36. Panahi Y, Hosseini MS, Khalili N, Naimi E, Simental-Mendia LE, Majeed M, Sahebkar A. Effects of curcumin on serum cytokine concentrations in subjects with metabolic syndrome: a post-hoc analysis of a randomized controlled trial. Biomed Pharmacother. 2016;82:578-82.

37. Heinl RE, Dhindsa DS, Mahlof EN, Schultz WM, Ricketts JC, Varghese T, Esmaeeli A, Allard-Ratick MP, Millard AJ, Kelli HM, et al. Comprehensive cardiovascular risk reduction and cardiac rehabilitation in diabetes and the metabolic syndrome. Can J Cardiol. 2016;32:S349-S57.

38. Brede S, Serfling G, Klement J, Schmid SM, Lehnert H. Clinical scenario of the metabolic syndrome. Visc Med. 2016;32:336-41.

39. Anand P, Kunnumakkara AB, Newman RA, Aggarwal BB. Bioavailability of curcumin: problems and promises. Mol Pharm. 2007;4:807-18.

40. Murdande SB, Pikal MJ, Shanker RM, Bogner RH. Aqueous solubility of crystalline and amorphous drugs: challenges in measurement. Pharm Dev Technol. 2011;16:187-200.

41. Mirzabeigi P, Mohammadpour AH, Salarifar M, Gholami K, Mojtahedzadeh M, Javadi MR. The effect of Curcumin on some of traditional and nontraditional cardiovascular risk factors: a pilot randomized, double-blind, Placebo-controlled Trial. Iran J Pharm Res. 2015;14:479-86.

42. Soare A, Weiss EP, Holloszy JO, Fontana L. Multiple dietary supplements do not affect metabolic and cardio-vascular health. Aging (Albany NY). 2014;6:149-57.

43. Neerati P, Devde R, Gangi AK. Evaluation of the effect of curcumin capsules on glyburide therapy in patients with type-2 diabetes mellitus. Phytother Res. 2014;28:1796-800
44. Jayakumar V, Ahmed SS, Ebenezar KK. Multivariate analysis and molecular interaction of curcumin with PPARgamma in high fructose diet induced insulin resistance in rats. Spring. 2016;5:1732

45. Nishiyama T, Mae T, Kishida H, Tsukagawa M, Mimaki Y, Kuroda M, Sashida Y, Takahashi K, Kawada T, Nakagawa K, Kitahara M. Curcuminoids and sesquiterpenoids in turmeric (Curcuma Longa L.) suppress an increase in blood glucose level in type 2 diabetic KK-ay mice. J Agric Food Chem. 2005; 53:959-63.

46. Kang Q, Chen A. Curcumin suppresses expression of low-density lipoprotein $(\mathrm{LDL})$ receptor, leading to the inhibition of $L D L$-induced activation of hepatic stellate cells. Br J Pharmacol. 2009;157:1354-67.

47. Sahebkar A. Are curcuminoids effective C-reactive protein-lowering agents in clinical practice? Evidence from a meta-analysis. Phytother Res. 2014;28:633-42.

48. Sahebkar A. Curcuminoids for the management of hypertriglyceridaemia. Nat Rev Cardiol. 2014;11:123.

49. Sahebkar A. Low-density lipoprotein is a potential target for curcumin: novel mechanistic insights. Basic Clin Pharmacol Toxicol. 2014;114:437-8.

50. Sahebkar A, Chew GT, Watts GF. Recent advances in pharmacotherapy for hypertriglyceridemia. Prog Lipid Res. 2014;56:47-66.

51. Panahi Y, Ahmadi Y, Teymouri M, Johnston TP, Sahebkar A. Curcumin as a potential candidate for treating hyperlipidemia: a review of cellular and metabolic mechanisms. J Cell Physiol. 2016; 10.1002/jcp.25756.

52. Cheng AL, Hsu CH, Lin JK, Hsu MM, Ho YF, Shen TS, Ko JY, Lin JT, Lin BR, Ming-Shiang W, et al. Phase I clinical trial of curcumin, a chemopreventive agent, in patients with high-risk or pre-malignant lesions. Anticancer Res. 2001;21:2895-900

\section{Submit your next manuscript to BioMed Central and we will help you at every step:}

- We accept pre-submission inquiries

- Our selector tool helps you to find the most relevant journal

- We provide round the clock customer support

- Convenient online submission

- Thorough peer review

- Inclusion in PubMed and all major indexing services

- Maximum visibility for your research

Submit your manuscript at www.biomedcentral.com/submit
) Biomed Central 